\title{
Chronic Fatigue Syndrome in Childhood Revisited: Not Common, But Not Unknown- Case Study and Literature Review
}

\author{
Alison Leong ( $\sim$ alisonleong8@gmail.com ) \\ Rajeev Ramachandran \\ National University Hospital, Singapore \\ Sajini Mary Varughese \\ National University Hospital, Singapore \\ Lee Gan Goh \\ National University Health System
}

National University Health System https://orcid.org/0000-0002-6180-4841

\section{Case report}

Keywords: Chronic fatigue syndrome, myalgic encephalomyelitis, adolescent

Posted Date: October 21st, 2021

DOI: https://doi.org/10.21203/rs.3.rs-962672/v1

License: (c) (i) This work is licensed under a Creative Commons Attribution 4.0 International License. Read Full License 


\section{Abstract}

Lethargy is a common non-specific complaint to primary care and has a broad range of differentials. Chronic fatigue syndrome (CFS) should be considered when there is persistent fatigue affecting function and post- exertional malaise six months or longer and initial workup is not suggestive of other organic causes; it is a debilitating condition that would benefit from diagnosis and treatment. We present an adolescent with CFS, the disease timeline, its impact and outcome.

\section{Introduction}

Fatigue is a common non-specific presentation to primary care with a broad range of differentials. A diagnosis of exclusion to consider would be chronic fatigue syndrome/ myalgic encephalomyelitis (CFS/ $\mathrm{ME})$, a debilitating condition. It commonly affects people 40-60 years of age but can also affect children, adolescents and adults of all ages. While adults may experience symptoms such as muscle and joint pain, children and adolescents may have headaches, abdominal pain and dizziness on standing / sitting up (1). We present a case of a teenage girl with CFS and the outcome.

\section{Definition}

CFS can be hard to diagnose as there are no specific tests, and symptoms may come and go and appear similar to other illnesses. The normality of blood tests and specific clinical criteria can help with diagnosis (2). Core symptoms from various criteria exist. This has been summarised by the CDC and include (3):

1. A reduced ability to carry out activities due to fatigue of six months or longer,

2. Post- exertional malaise comprising worsening of CFS symptoms after a physical or mental activity that would not have caused a problem prior to illness,

3. Sleep disturbances, not feeling better or less tired even after a full night's sleep. In children this may manifest as reduced energy levels, daytime sleepiness, difficulty falling or staying asleep, vivid dreams, and

4. One or both of:

a) thinking or memory problems, or

b) worsening of symptoms while standing or sitting upright which may include feeling lightheaded, dizzy, weak, having vision changes.

Other common symptoms include muscle pain, joint pain without swelling or redness, new or worsening of headaches. Children more commonly experience headaches or abdominal pain rather than muscles aches or joint pains. 
Some patients with CFS may also have tender lymph node in neck or arm, sore throat, digestive issues like irritable bowel syndrome, chills and night sweats, allergies and sensitivities to foods, odours, chemicals, light, or noise, muscle weakness, shortness of breath or irregular heartbeat as a result of association with central sensitisation (3).

It is important to find out how the disease affects a patient's daily functioning. Questions to ask include what the patient is able to do now compared to what they were able to do before, how long they have felt this way, what makes them feel better or worse, what happens when they try to do activities that are hard for them now, symptoms keeping them from doing what they want to do and whether they have trouble remembering or focusing on tasks (1).

\section{Case Presentation}

\section{History}

A 15-year-old girl presented to General Paediatrics with lethargy, described as sleeping 16-18 hours a day for the last seven months and a decrease in function where she was unable do strenuous activities though previously being active in sports (see Figure 1). She also started to miss school and a meal each day when she was sleeping. She had coryzal symptoms and sore throat in the first two months. Along with that she had a bitemporal headache that started seven months ago and was improving with no associated nausea or vomiting. For the last 2 months she had epigastric pain occurring daily that occurred after treatment with Clarithromycin. For the last six weeks she also experienced near syncope with postural dizziness and darkening of vision on getting up. When awake, she was able to study, eat and go for walks though of a less strenuous nature. Patient had good sleep hygiene and uninterrupted sleep for 16-18 hours a day.

There were no autoimmune features such as frequent fevers, rashes, joint swelling or pains, no $B$ symptoms such as weight loss or night sweats, no features of malignancy such as bone pain, bleeding diathesis, or anaemia. There were no other infective symptoms such as vomiting or diarrhoea, or urinary tract symptoms and her coryzal symptoms in the first two months were attributed to chronic rhinitis. There were no "slapped checks" or reticular rash or lymphadenopathy or sore throat or cough. There was no significant medication, social, sexual or travel history. She denied a low mood and had no stressors with life, and had no suicidal ideation or thoughts of self-harm.

\section{Family}

Patient stays with her biological parents and two siblings. She has good family support.

\section{Physical Findings}

Patient's blood pressure was $105 / 64$, heart rate $74 /$ min which on standing was $115 / 63,103 / \mathrm{min}$. Physical examination was normal. Body mass index was 19.9 .

\section{Provisional diagnoses}


Provisional diagnosis was chronic fatigue syndrome (CFS). The patient met the following criteria for CFS, namely the duration of fatigue, significantly limited activity levels since onset of illness with the patient premorbidly being active in sport, post exertional tiredness, non- refreshing sleep despite sleeping 16 hours, and associated symptoms of headaches, abdominal pain and orthostatic symptoms. There were no other causes to be found such as depression, endocrine, autoimmune causes, malignancy, chronic infections or congestive cardiac failure, chronic liver disease and chronic renal failure.

\section{Investigations}

Prior to presentation she had recurrent visits to general practitioners and to Ear, Nose and Throat specialists. Laboratory tests done such as a full blood count, fasting glucose, a renal and electrolytes and liver panel and a thyroid function test, and C- reactive protein were normal. Urinalysis was normal.

\section{Subsequent investigations}

Patient had a previous admission where further labs were done. Serum cortisol and ACTH, and hepatitis panel were normal. Calcium, phosphate, vitamin B12, vitamin D, ferritin and folic acid was normal. An Antistreptolysin $\mathrm{O}$ Titer test as well as EBV IgM test was negative. A syphilis test was non-reactive and $\mathrm{HIV}$ test was negative. A urea breath test for $\mathrm{H}$. Pylori was negative. MRI brain done showed no gross intracranial abnormality. Frontal, ethmoid, sphenoid sinuses were well pneumatised with no significant air fluid levels.

\section{Timeline of illness}

\section{Management and progress}

Patient and parents were counselled on the nature of chronic fatigue syndrome, and that the goal was to reduce symptoms of illness that may wax and wane and improve quality of life. Patient was referred to Occupational and Physiotherapist for activity pacing, home activity scheduling and sleep hygiene with the aim of transitioning back to school.

Over the course of a year, patient became less tired and returned back to her usual hours of sleep (figure 1). Her mood was stable. However, she was still not at her premorbid condition. She was able to restart physical activities such as playing frisbee once a week and basketball twice a week. However, she was not able to return to a competitive level of sports / running as she felt tired after and she felt breathless wearing masks. Attempts were made to reintegrate back to school, however patient was not able to attend lessons and exams due to tiredness especially after physical or mental effort. Patient hence started home schooling which allowed for individualisation of teaching schedule based on her energy levels in preparation for a major exam.

\section{Discussion}

\section{Prognosis and time course}


A Pubmed search on a CFS prognosis was done with the search terms ("chronic fatigue syndrome" OR "Myalgic Encephalomyelitis") AND prognosis AND (young OR pediatric OR paediatric OR teenager OR adolescent) revealing 1,350 results. Titles and abstracts were screened and the relevant articles full text were read of which nine stated the course or prognosis of CFS (4-12). References in relevant articles were also screened revealing two additional articles $(13,14)$.

CFS can begin suddenly or gradually with a spectrum of severity of loss in physical and cognitive functioning, with mildly affected patients being able to attend school full time or part time with a limitation in sport and after school activities and school absences(7). Severely affected patient may be homebound or wheelchair bound with inability to participate in home tutoring sessions (7). Affected adolescents can make a full recovery with time, patience, explanation of factors contributing to illness, and managing setbacks (4).

A thirteen year follow up of 35 children and adolescents found that the average age of disease onset was 12 and that $37.1 \%$ considered themselves resolved of illness; $42.9 \%$ considered themselves well but not resolved; $11.4 \%$ considered themselves chronically ill; and $8.6 \%$ considered themselves more ill than before (14). Eight participants (22.9\%) missed more than two years of school (14). The impact of the illness was most evident in terms of education (6).

In another study of 25 adolescents with CFS, $68 \%$ were unable to attend school with a mean time of 1 year out of school (8). The mean duration of illness was 37.5 months (8).

An observational study of 784 young people attending a paediatric outpatient service found that the mean duration of illness was 5 years with $38 \%$ and $68 \%$ of patients reporting recovery by 5 and 10 years respectively, with depression, anxiety and severity of illness being non predictive of recovery (9).

In contrast, a systematic review of 26 studies found an older age, more chronic illness, having a comorbid psychiatric disorder and holding a belief that the illness is due to physical causes are factors for poor prognosis(13).

A longitudinal study of 13,978 children found that $75 \%$ of adolescents with chronic disability fatigue recovered after two to three years (10).

\section{Pathophysiology and triggers}

CFS is proposed to be caused by an immune system dysfunction, hypothalamic pituitary, or adrenal gland dysfunction, or central and autonomic nervous system abnormalities. Predisposing factors include being female in post pubertal adolescents with prevalence being $3-4$ times higher than in boys, and also a family history of CFS (7). Viral infection, such as Epstein-Barr virus or human herpesvirus 6 infection are possible precipitating factors (15)(16). In some patients no physical or emotional trauma, over exertion or no precipitating factors have been identified (7). Cytokine expressions have been implicated in CFS; the "cytokine storm" exhibited by some COVID-19 patients has been hypothesized to 
lead to post infectious fatigue (17). Perpetuating factors include a late diagnosis, overexertion resulting in "crashes," stress, inadequate sleep (7).

A pubmed search for case reports on CFS with the following search terms (chronic fatigue syndrome) NOT adult AND (evidence) NOT (covid-19) NOT (protocol)) AND (case study) revealed two articles (18) (19). The first showed elevation of choline concentration in the brain of three patients with CFS indicating abnormal cerebral blood flow. The second was a case report showing parvovirus infection should be excluded in patients with CFS.

\section{Evaluation}

A careful history taking including precipitating factors, sleep disturbances and psychosocial stressors is needed. OSA screening can be done e.g. using STOP-BANG questionnaire. Red flag symptoms to rule out include chest pain, dyspnoea, lymphadenopathy, weight loss, inflammatory signs or joint pain, and focal neurological deficits. These may point towards cardiac, pulmonary disease, malignancy, autoimmune disease, and central nervous system malignancy/ abscess/ multiple sclerosis (20).

Examination may include signs of low blood pressure or orthostatic hypotension, tachycardia, a positive rhomberg sign, low oral temperature or slightly elevated body temperature $<37.7$ degrees (15).

Testing is done to confirm or exclude other causes of fatigue or syndromes (see section on differentials below). These may include a full blood count, glucose, calcium, electrolytes, renal and liver function tests, and thyroid function tests. Depending on the symptoms/ signs, other tests such as evaluation for adrenal insufficiency, creatinine kinase for muscle pain or weakness, or sleep study is performed if sleep apnoea is suspected. Neuroimaging is not routinely performed.

In the primary care setting, patients are periodically followed up and laboratory investigations done as required. Comorbidities such as anxiety and depression identified. Although a diagnosis requires at least six months of illness, this time period should include follow up appointments, complete investigations and allow time for improvement for children with illnesses with similar symptoms as CFS, but do not usually last as long as CFS (1). Management should also occur during this period. A referral to a rheumatologist, neurologist or sleep specialist can made as necessary.

\section{Differentials}

The fact that there are many diseases with lethargy as a symptom makes it essential to rule them out before reaching a diagnosis. These include autoimmune disease such as SLE and juvenile rheumatoid arthritis, endocrine disorders such as diabetes and hypothyroidism, congestive cardiac failure, chronic liver disease and chronic renal failure, neuromuscular disease such as multiple sclerosis and myasthenia gravis, malignancies, leukaemia, chronic infection with tuberculosis, hepatitis B/C, HIV/ AIDS and localised infection such as sinusitis. Other causes include parasitic disease such as giardiasis and toxoplasmosis, sleep disorders such as sleep apnoea, narcolepsy, medication side effects, substance abuse and psychiatric disorders (21). 
Postural tachycardia syndrome (POTS) involving orthostatic tachycardia without orthostatic hypotension is often found comorbid with CFS. It involves a heart rate increase of greater than 30 beats per minute when patients go from laying down to standing in the absence of other causes of orthostatic hypotension, such as dehydration.

There are increased rates of psychiatric disorders in young people with CFS, with depressive and anxiety disorders being the most commonly reported comorbid problems (21). Central Sensitivity Syndromes involve syndromes sharing the common mechanism of a heightened perception of pain and sometimes triggering abnormal responses. These include CFS, irritable bowel syndrome, chronic headaches and fibromyalgia.

\section{Treatment}

Once a diagnosis is made, the physician should provide information on the possible causes and course of CFS.

For example, precipitating factors identified such as physical, infectious and psychological causes and perpetuating factors such as poor sleep, reduced activity causing deconditioning, stress, over exertion.

The patient should be advised that setbacks/relapses are to be expected and may be triggered by unexpected/unplanned activities, poor sleep, infection or stress. A shared decision making should be made regarding the pace of interventions. Engagement with the family is important for young patients.

Treatment plan is individualised based on symptoms, support and educational needs. General management would involve management of symptoms such as pain and dizziness, sleep management, pacing, rest periods, and adjustments to studies or work to help patients return when they are fit enough (22).

Behavioural approaches to help children regulate their activities and improve sleep include Cognitive Behavioural Therapy which challenges unhelpful thoughts about their symptoms, Graded Exercise Therapy (GET) focusing on use of regular physical activity, starting from a little and increasing the duration and intensity over time and Activity Management involving splitting activities into achievable tasks followed by increasing in complexity (23).

A self-modified activity pacing involving patient estimation of current physical and mental capabilities before commencing desired activities in personal care, productivity and leisure as well as graded exercise therapy once patient can control their daily life activities without excessive feelings of fatigue was shown to improve satisfaction and performance in daily life activities (24).

Early in the illness, most children may not be able to attend school. An awareness of how the schools that their patient attend functions is important. For example, as different teachers are scheduled to teach at different times each day, recommending patients to attend at a fixed time daily would not be feasible as it would mean encountering different subjects each day. Instead, it could be better to reduce subjects 
depending on the child's career goals, subject preferences and what is needed to progress through schools. Of importance is feeling understood and believed by teachers and doctors about their condition (25).

A plan is needed to be in place for managing setbacks as a result of "push-and-crash" cycles which may involve relaxation and breathing techniques, trying to maintain activity and exercise levels by pacing activities/ alternating activities with breaks. However, in some setbacks/ relapses, it may be required to stop activity and increase rest period to stabilise symptoms and re-establish a new baseline (22). There is a need to plan activity and rest to stay within limits of tolerance or "energy envelope."

\section{Conclusion}

CFS/ ME should be considered as a possible diagnosis once other diseases have been ruled out. The non-specific nature of this illness may make it difficult to suspect this diagnosis. An appropriate diagnosis allows for proper care from the physician and accommodation from the school system. The initial diagnosis, management and follow up requires specialist follow up providing individualised care. A multidisciplinary team involving occupational therapy, physiotherapy and psychotherapy may be needed. The greatest impact in young patients would be on education, and the clinician needs to be sensitive to the relationship between the young person, her/his parents, and the school as many families have followed a long and circuitous route to a diagnosis and the patient may be lagging behind months in school, and a personalised education plan that flexibly accommodates their illness created if possible (7). Follow up needs to be continued until the patient is well and able to function normally.

\section{Declarations}

\section{Ethics approval and consent to participate}

Not applicable

\section{Consent for publication}

Obtained from patient and parent

\section{Availability of data and material}

Not applicable

\section{Competing interests}

Not applicable

\section{Funding}


Not applicable

\section{Authors' contributions}

Writing of case report, Dr Alison Leong (Family Medicine Resident at National University Health System, Singapore; Feedback and comments from Dr Sajini Mary Varughese (Consultant, Paediatrics, National University Hospital, Singapore) and Dr Rajeev Ramachandran (Senior Consultant, Paediatrics, National University Hospital, Singapore); Supervisor, A/Prof Goh Lee Gan (Senior Consultant, Department of Family Medicine, National University Health System)

\section{Acknowledgements}

Not applicable

\section{References}

1. CDC. Symptoms and Diagnosis of ME_CFS in Children _ Myalgic Encephalomyelitis_Chronic Fatigue Syndrome (ME_CFS) _ CDC [Internet]. Available from: https://www.cdc.gov/me-cfs/me-cfschildren/children-symptoms-diagnosis.html.

2. Bansal AS. Investigating unexplained fatigue in general practice with a particular focus on CFS/ME. BMC Fam Pract [Internet]. 2016;17(1):1-14. Available from: http://dx.doi.org/10.1186/s12875-0160493-0.

3. CDC. Myalgic Encephalomyelitis / Chronic Fatigue Syndrome Symptoms of ME / CFS Primary Symptoms [Internet]. 2020. p. 1-2. Available from: https://www.cdc.gov/me-cfs/symptomsdiagnosis/symptoms.html.

4. Burgess $M$, Chalder T. Adolescents with severe chronic fatigue syndrome can make a full recovery. BMJ Case Rep. 2011;2-5.

5. Brown MM, Bell DS, Jason LA, Christos C, Bell DE. Understanding long-term outcomes of chronic fatigue syndrome. J Clin Psychol [Internet]. 2012/06/29. 2012 Sep;68(9):1028-35. Available from: https://pubmed.ncbi.nlm.nih.gov/22753044.

6. Patel MX, Smith DG, Chalder T, Wessely S. Chronic fatigue syndrome in children: A cross sectional survey. Arch Dis Child. 2003;88(10):894-8.

7. Rowe PC, Underhill RA, Friedman KJ, Gurwitt A, Medow MS, Schwartz MS, et al. Myalgic encephalomyelitis/chronic fatigue syndrome diagnosis and management in young people: A primer. Front Pediatr. 2017;5(June).

8. Society R. The course of severe chronic fatigue syndrome in childhood. J R Soc Med [Internet]. 2000;129-34. Available from: http://www.ncbi.nlm.nih.gov/pmc/articles/PMC1297949/pdf/10741312.pdf.

9. Rowe KS. Long term follow up of young people with chronic fatigue syndrome attending a pediatric outpatient service. Front Pediatr. 2019;7(FEB):1-18. 
10. Norris T, Collin SM, Tilling K, Nuevo R, Stansfeld SA, Sterne JAC, et al. Natural course of chronic fatigue syndrome/myalgic encephalomyelitis in adolescents. Arch Dis Child. 2017;102(6):522-8.

11. Chu L, Valencia IJ, Garvert DW, Montoya JG. Onset patterns and course of myalgic encephalomyelitis/chronic fatigue syndrome. Front Pediatr. 2019;7(FEB):1-22.

12. Jason L, Barker K, Brown A. Pediatric Myalgic Encephalomyelitis/Chronic Fatigue Syndrome. Rev Heal Care. 2012;3(4):257-70.

13. Joyce J, Hotopf M, Wessely S, Joyce J, Hotopf MWS. The prognosis of chronic fatigue and chronic fatigue syndrome: a systematic review. QJM. 1997 Mar;90(3):223-33.

14. MD DSB, Jordan K, Robinson M. Thirteen-Year Follow-Up of Children and Adolescents With Chronic Fatigue Syndrome. Pediatrics [Internet]. 2001 May 1;107(5):994 LP - 998. Available from: http://pediatrics.aappublications.org/content/107/5/994.abstract.

15. Khouzam HR. Chronic fatigue syndrome: An update on diagnosis in primary care [Internet]. Vol. 51, Patient Care Online. 2011. Available from: https://www.patientcareonline.com/view/chronic-fatiguesyndromean-update-diagnosis-primary-care.

16. Rivera MC, Mastronardi C, Silva-aldana CT, Arcos-burgos M, Lidbury BA. Myalgic Encephalomyelitis / Chronic Fatigue Syndrome: A Comprehensive Review. Diagnostics. 2019;9(91):1-34.

17. Islam MF, Cotler J, Jason LA. Post-viral fatigue and COVID-19: lessons from past epidemics. Fatigue Biomed Heal Behav [Internet]. 2020;8(2):61-9. Available from:

https://doi.org/10.1080/21641846.2020.1778227.

18. Tomoda A, Miike T, Yamada E, Honda H, Moroi T, Ogawa M, et al. Chronic fatigue syndrome in childhood. Brain Dev. 2000 Jan;22(1):60-4.

19. Jacobson SK, Daly JS, Thorne GM, Mclntosh K. Chronic parvovirus B19 infection resulting in chronic fatigue syndrome: case history and review. Clin Infect Dis an Off Publ Infect Dis Soc Am. 1997 Jun;24(6):1048-51.

20. Yancey JR, Thomas SM. Chronic fatigue syndrome: Diagnosis and treatment. Am Acad Fam Physicians. 2012;86(8):741-6.

21. Jordan KM, Landis DA, Downey MC, Osterman SL, Thurm AE, Jason LA. Chronic Fatigue Syndrome in Children and Adolescents: A Review. J Adolesc Heal. 1998;22(4):4-18.

22. NICE. Chronic fatigue syndrome / myalgic encephalomyelitis (or encephalopathy): Diagnosis and Management (NICE) [Internet]. National Institute for Health and Care Excellence. 2007. Available from: https://www.nice.org.uk/guidance/cg53.

23. Crawley E. Pediatric chronic fatigue syndrome: current perspectives. Pediatr Heal Med Ther. 2018; Volume 9:27-33.

24. Kos D, Eupen I, Van, Meirte J, Cauwenbergh D. Van. Activity Pacing Self-Management in Chronic Fatigue. Am J Occup Ther. 2015;69(5):1-11.

25. Rowe K. Paediatric patients with myalgic encephalomyelitis/chronic fatigue syndrome value understanding and help to move on with their lives. Acta Paediatr Int J Paediatr. 2020;109(4):790- 


\section{Figures}

\section{Onset of illness:}

\section{Coryzal}

symptoms with

sore throat

followed by

fatigue. Slept 16

to 18 hours a

day. Missed

school.
Months 3 to 4:

Month 5:

Improvement to

sleeping 8 to 10

hours a day, able to Improvement of but still felt tired. headache and fatigue.

Returned back to school.
Went for overseas travel in later half of month but fatigue recurred.

\section{Month 6:}

Recurrence

of fatigue.

Sleeping 16

to 18 hours a

day. Also had

postural

dizziness and

near

syncopal

episodes.
Month 7:

Improvement of fatigue, sleeping

12 hours a day.

Able to engage

in light physical

activities.
Months $\mathbf{8}$ to 22: Went back to school however unable to attend most classes, missed a major examination. Decision made to switch to home schooling. Fatigue has improved, back to sleeping 8 hours a day. Able to play basketball twice a week but not do strenuous activities she premorbidly was able to do.

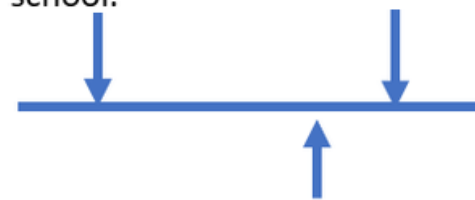

Months 2 to 3:

Multiple visits to General Practitioners then ENT where blood tests done. Was treated for sinusitis.
Month 6: Admitted and treated as for post viral fatigue syndrome.

\section{Figure 1}

Timeline of patient's progress. 